This item was submitted to Loughborough's Research Repository by the author.

Items in Figshare are protected by copyright, with all rights reserved, unless otherwise indicated.

\title{
Partition of mixed-mode fractures in 2D elastic orthotropic laminated beams under general loading
}

\section{PLEASE CITE THE PUBLISHED VERSION}

http://dx.doi.org/10.1016/j.compstruct.2016.04.016

\section{PUBLISHER}

(C) Elsevier

\section{VERSION}

AM (Accepted Manuscript)

\section{PUBLISHER STATEMENT}

This work is made available according to the conditions of the Creative Commons Attribution-NonCommercialNoDerivatives 4.0 International (CC BY-NC-ND 4.0) licence. Full details of this licence are available at: https://creativecommons.org/licenses/by-nc-nd/4.0/

\section{LICENCE}

CC BY-NC-ND 4.0

\section{REPOSITORY RECORD}

Wood, Joe, Christopher Harvey, and Simon Wang. 2016. "Partition of Mixed-mode Fractures in 2D Elastic Orthotropic Laminated Beams Under General Loading”. figshare. https://hdl.handle.net/2134/21038. 


\title{
Partition of mixed-mode fractures in 2D elastic orthotropic laminated beams under general loading
}

\author{
J. D. Wood, C. M. Harvey*, S. Wang \\ Department of Aeronautical and Automotive Engineering, Loughborough University, \\ Loughborough, Leicestershire LE11 3TU, United Kingdom
}

\begin{abstract}
An analytical method for partitioning mixed-mode fractures on rigid interfaces in orthotropic laminated double cantilever beams (DCBs) under through-thickness shear forces, in addition to bending moments and axial forces, is developed by extending recent work by the authors (Harvey et al., 2014). First, two pure through-thickness-shear-force modes (one pure mode I and one pure mode II) are discovered by extending the authors' mixed-mode partition theory for Timoshenko beams. Partition of mixed-mode fractures under pure through-thickness shear forces is then achieved by using these two pure modes in conjunction with two thickness ratio-dependent correction factors: (1) a shear correction factor, and (2) a pure-mode-II energy release rate (ERR) correction factor. Both correction factors closely follow an elegant normal distribution around a symmetric DCB geometry. The principle of orthogonality between all pure mode I and all pure mode II fracture modes is then used to complete the mixed-mode fracture partition theory for a general loading condition, including bending moments, axial forces, and through-thickness shear forces. Excellent agreement is observed between the present analytical partition theory and numerical results from finite element method (FEM) simulations.
\end{abstract}

Keywords: Energy release rate, Fracture mode partitioning, Orthotropic laminated composites, Mixed-mode fracture, Orthogonal pure modes, Shear forces

* Corresponding Author 


\section{Introduction}

An analytical method for partitioning mixed-mode fractures in orthotropic laminated double cantilever beams (DCBs) with rigid interfaces has been developed in the authors' recent work [1] by taking 2D elasticity into consideration in a novel way. In Ref. [1], the DCBs are under crack tip bending moments and axial forces. The present study extends Ref. [1] to include crack tip throughthickness shear forces which commonly occur in practice.

Some of the previous studies on the topic include Lu et al.'s work [2] where the finite element method (FEM) was used to investigate the effects of transverse shear on an orthotropic beam with a crack present. Orthotropic rescaling simplified the numerical analysis to three non-dimensional parameters. The total energy release rate (ERR) was obtained by using the J-integral and then the crack tip displacements allowed the total ERR to be partitioned into its individual mode I and II components. Wang and Qiao [3] extended the conventional 2D elasticity-based partition theory of Suo and Hutchinson [4] to take into account shear deformation by using first-order sheardeformable plate theory for a bimaterial interfacial crack. The total ERR was determined using the J-integral and related to the individual stress intensity factors by introducing an unknown parameter which, as the solution to an integral equation, must be determined from tabulated numerical data from a limited range of geometries and material configurations. Li et al. [5], also using Suo and Hutchinson's work [4], considered the effects of transverse shear loading on a crack between a layered, isotropic, linear elastic material. They asserted that it is not possible to use beam theories (even higher-order ones) to determine the shear component of the ERR because such an approach neglects the contribution of the deformation local to the crack-tip, which plays a crucial role. They concluded that a full elastic solution is required and used the FEM. They combined their results with Suo and Hutchinson's theory [4] for bending moments and axial forces to provide the ERR and mode partition for layered materials under general loading conditions. A disadvantage of this method, again, is its reliance on tabulated data. The effect of through-thickness shear forces on the fracture-mode partition of a DCB therefore still remains a crucial area of research.

The authors' partition theory based on classical beam theory [6-10] has given excellent predictions of mixed-mode fracture toughness for delamination in generally laminated composite beams [8-10] when compared against the experimental test data from some independent comprehensive testing [11-20]. In comparison, mixed-mode partition theories based on 2D elasticity [1,4] have shown poor correlation with experimental results. It is, however, still an unanswered question as to which mixed-mode partition theory provides the most accurate results for brittle fracture subjected to other loading conditions such as fatigue or thermal loading. Therefore, it is still essential to develop mixed-mode partition theories based on 2D elasticity in 
order to provide a comprehensive set of tools for the understanding of interfacial fractures. This is the motivation of present work.

The format of this paper is as follows: Initially, in Section 2.1, the ERR partition based on Timoshenko beam theory [6-10] is extended to consider a 2D elastic orthotropic DCB with through-thickness shear forces alone at the crack tip. Then, in Section 2.2, a mixed-mode partition theory is established for the DCB under general loading conditions which include crack tip bending moments, axial forces and shear forces. Comparisons for the mixed-fracture mode partition theory are made against results from 2D FEM simulations for a combination of loading conditions in Section 3. Conclusions are drawn in Section 4.

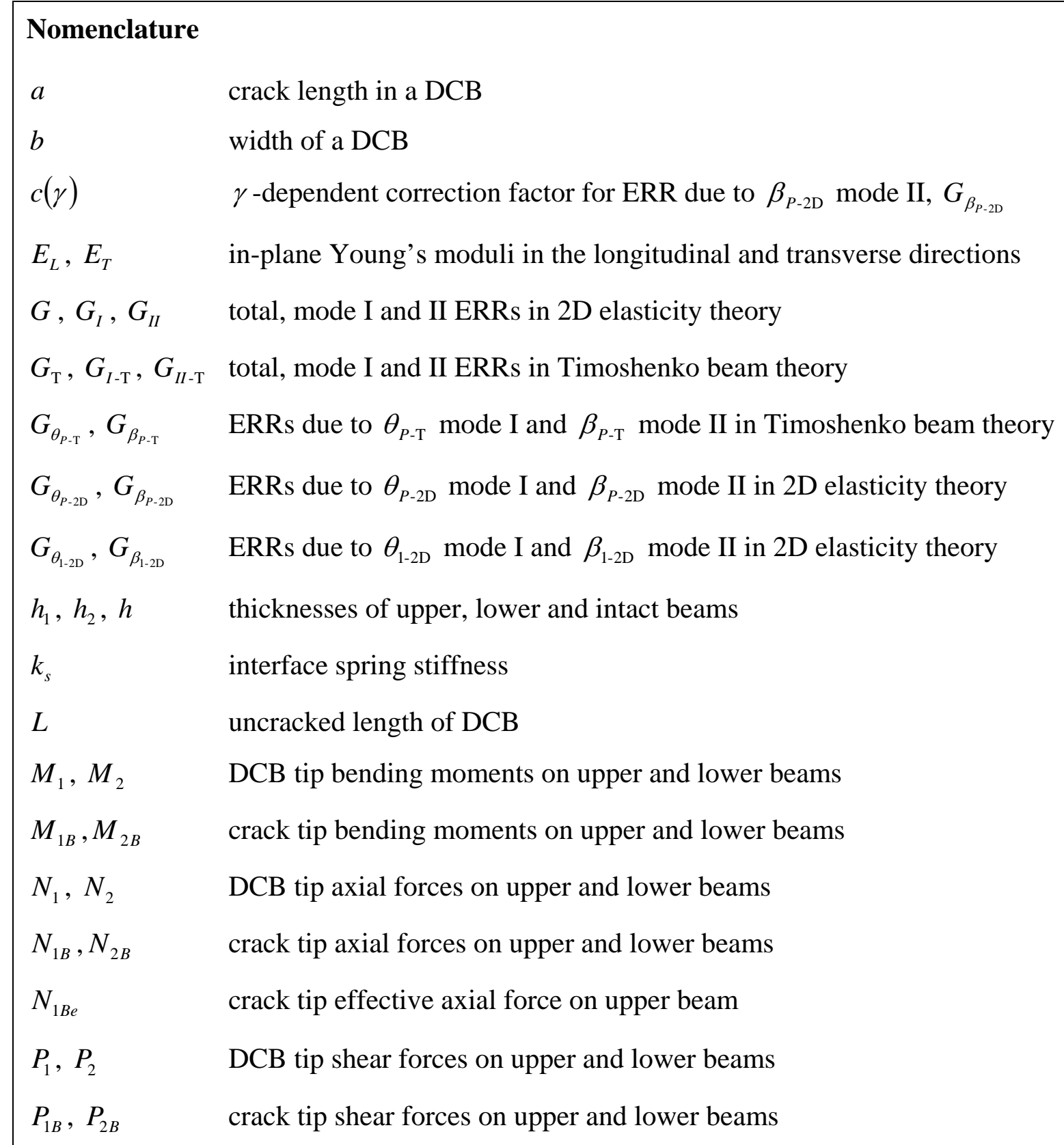




\begin{tabular}{|ll|}
\hline$\gamma$ & thickness ratio, $h_{2} / h_{1}$ \\
$\theta_{i-2 \mathrm{D}}, \beta_{i-2 \mathrm{D}}$ & pure mode I and II (with $i=1,2,3,4)$ in 2D elasticity theory \\
$\theta_{P-\mathrm{T}}, \beta_{P-\mathrm{T}}$ & shear force only pure mode I and II in Timoshenko beam theory \\
$\theta_{P-2 \mathrm{D}}, \beta_{P-2 \mathrm{D}}$ & shear force only pure mode I and II in 2D elasticity theory \\
$\kappa(\gamma)$ & $\gamma$-dependent through-thickness shear correction factor \\
$\mu_{L Z}$ & through-thickness shear modulus \\
$v_{L T}, v_{T L}$ & in-plane Poisson's ratios \\
$\sigma_{n}, \tau_{s}$ & interface normal and shear stresses \\
& \\
Abbreviations & \\
DCB & double cantilever beam \\
ERR & energy release rate \\
FEM & finite element method \\
VCCT & virtual crack closure technique \\
\hline
\end{tabular}

\section{Analytical development}

2.1. Mixed-mode partitions with through-thickness shear forces alone at the crack tip

Fig. 1a shows a laminated DCB with its geometry and tip bending moments $M_{1}$ and $M_{2}$, axial forces $N_{1}$ and $N_{2}$, and through thickness shear forces $P_{1}$ and $P_{2}$. Fig. 1b shows the internal loads at the crack tip and the sign convention of the interface normal stress $\sigma_{n}$ and shear stress $\tau_{s}$. The total ERR and its partitions under crack tip through-thickness shear forces, $P_{1 B}$ and $P_{2 B}$, are calculated from Timoshenko beam partition theory [6-10] as:

$$
\begin{gathered}
G_{\mathrm{T}}=\frac{1}{2 b^{2} h_{1} \kappa \mu_{L Z}}\left(P_{1 B}{ }^{2}+\frac{P_{2 B}{ }^{2}}{\gamma}-\frac{\left(P_{1 B}+P_{2 B}\right)^{2}}{1+\gamma}\right)=\left\{\begin{array}{ll}
P_{1 B} & P_{2 B}
\end{array}\right\}\left[C_{\mathrm{T}}\right]\left\{\begin{array}{ll}
P_{1 B} & P_{2 B}
\end{array}\right\}^{T} \\
G_{I-\mathrm{T}}=C_{I-\mathrm{T}}\left(P_{1 B}-\frac{P_{2 B}}{\beta_{P-\mathrm{T}}}\right)^{2} \\
G_{I I-\mathrm{T}}=C_{I I-\mathrm{T}}\left(P_{1 B}-\frac{P_{2 B}}{\theta_{P-\mathrm{T}}}\right)^{2}
\end{gathered}
$$

where 


$$
\begin{aligned}
& c_{I-\mathrm{T}}=G_{\theta_{P-\mathrm{T}}}\left(1-\frac{\theta_{P-\mathrm{T}}}{\beta_{P-\mathrm{T}}}\right)^{-2} \quad, \quad c_{I I-\mathrm{T}}=G_{\beta_{P-\mathrm{T}}}\left(1-\frac{\beta_{P-\mathrm{T}}}{\theta_{P-\mathrm{T}}}\right)^{-2} \\
& G_{\theta_{P-\mathrm{T}}}=\frac{1}{2 b^{2} h_{1} \kappa \mu_{L Z}}\left(1+\frac{\theta_{P-\mathrm{T}}^{2}}{\gamma}-\frac{\left(1+\theta_{P-\mathrm{T}}\right)^{2}}{1+\gamma}\right) \\
& G_{\beta_{P-\mathrm{T}}}=\frac{1}{2 b^{2} h_{1} \kappa \mu_{L Z}}\left(1+\frac{\beta_{P-\mathrm{T}}{ }^{2}}{\gamma}-\frac{\left(1+\beta_{P-\mathrm{T}}\right)^{2}}{1+\gamma}\right) \\
& {\left[C_{\mathrm{T}}\right]=\frac{1}{2 b^{2} h_{1} \kappa \mu_{L Z}(1+\gamma)}\left[\begin{array}{cc}
\gamma & -1 \\
-1 & \gamma^{-1}
\end{array}\right]}
\end{aligned}
$$

and $\gamma=h_{2} / h_{1}$ is the thickness ratio, $b$ is the width of the beam, and $\mu_{L Z}$ is the through-thickness shear modulus with the correction factor $\kappa$, usually taken to be $5 / 6$. The ratios $\left(\theta_{P-\mathrm{T}}, \beta_{P \text {-T }}\right)=(-1, \gamma)$ represent the set of orthogonal pure modes. The subscript $P$ - T denotes that the pure modes are for the through-thickness shear forces at the crack tip, $P_{1 B}$ and $P_{2 B}$, and are based on Timoshenko beam theory. That is, when $P_{2 B}=\theta_{P-\mathrm{T}} P_{1 B}$ pure mode I occurs. Its orthogonal pure mode II is $\beta_{P-\mathrm{T}}$. Similarly, when $P_{2 B}=\beta_{P-\mathrm{T}} P_{1 B}$ the pure mode II mode occurs. Here, the 'orthogonal' means

$$
\left\{\begin{array}{ll}
1 & \beta_{P-\mathrm{T}}
\end{array}\right\}\left[C_{\mathrm{T}}\right]\left\{\begin{array}{ll}
1 & \theta_{P-\mathrm{T}}
\end{array}\right\}^{T}=0
$$

It is worth noting that $G_{I I-T}=0$ as $G_{\beta_{P-\mathrm{T}}}=0$ with $\beta_{P-\mathrm{T}}=\gamma$. That is, the two crack tip throughthickness shear forces, $P_{1 B}$ and $P_{2 B}$, only produce mode I ERR within the context of the Timoshenko beam theory. Obviously, this will not be the case within the context of 2D elasticity. The total ERR $G$ is expected to be different from the $G_{\mathrm{T}}$ in Eq. (1) as the constant throughthickness shear correction factor $\kappa=5 / 6$ is no longer valid. Moreover, the orthogonal pure mode set $\left(\theta_{P-\mathrm{T}}, \beta_{P-\mathrm{T}}\right)=(-1, \gamma)$ will also change to be $\left(\theta_{P-2 \mathrm{D}}, \beta_{P-2 \mathrm{D}}\right)$. Interestingly, it is found from FEM simulations that

$$
\left(\theta_{P-2 \mathrm{D}}, \beta_{P-2 \mathrm{D}}\right)=\left(-1, \gamma \exp \left(-1.986060 \operatorname{atanh}\left(0.563483 \gamma_{i}\right)\right)\right)
$$

where $\gamma_{i}=\log _{10}(1 / \gamma)$. Note that the $\theta_{P-2 \mathrm{D}}$ pure mode remains the same as $\theta_{P \text {-T }}=-1$ and that the $\beta_{P-2 \mathrm{D}}$ pure mode has an exponential multiplier on $\beta_{P-\mathrm{T}}=\gamma$. It is easily shown that $\beta_{P-2 \mathrm{D}}(1 / \gamma)=1 / \beta_{P-2 \mathrm{D}}(\gamma)$ as required by physical symmetry. Fig. 2 shows the variation of the puremode-II $\beta_{P-2 \mathrm{D}}$ mode with respect to $\gamma$. Eq. (9), for the pure-mode-II $\beta_{P-2 \mathrm{D}}$ mode, is only valid in the range where $-1.7 \leq \gamma_{i} \leq 1.7$. It was not possible to determine $\beta_{P-2 \mathrm{D}}$ using the FEM outside this 
range due to the very large numbers of finite elements required. The pure-mode-I $\theta_{P-2 \mathrm{D}}$ mode remains unchanged.

Note that $\beta_{P-2 \mathrm{D}} \approx \gamma^{1.5}$ is an accurate approximation if $\gamma \approx 1$. At $\gamma=3$ or $\gamma=1 / 3$ it approximates $\beta_{P-2 D}$ to within about $1 \%$ of the actual value. Inside this range the accuracy is much higher; however, outside it the accuracy decreases rapidly and Eq. (9) should be used instead.

By using the pure mode set in Eq. (9), the ERR partitions based on 2D elasticity, $G_{I}$ and $G_{I I}$, can be written as

$$
\begin{aligned}
& G_{I}=C_{I P}\left(P_{1 B}-\frac{P_{2 B}}{\beta_{P-2 \mathrm{D}}}\right)^{2} \\
& G_{I I}=C_{I I P}\left(P_{1 B}-\frac{P_{2 B}}{\theta_{P-2 \mathrm{D}}}\right)^{2}
\end{aligned}
$$

where

$$
\begin{gathered}
c_{I P}=G_{\theta_{P-2 \mathrm{D}}}\left(1-\frac{\theta_{P-2 \mathrm{D}}}{\beta_{P-2 \mathrm{D}}}\right)^{-2}, \quad c_{I I P}=G_{\beta_{P-2 \mathrm{D}}}\left(1-\frac{\beta_{P-2 \mathrm{D}}}{\theta_{P-2 \mathrm{D}}}\right)^{-2} \\
G_{\theta_{P-2 \mathrm{D}}}=\frac{1}{2 b^{2} h_{1} \bar{E} \kappa(\gamma)}\left(1+\frac{\theta_{P-2 \mathrm{D}}^{2}}{\gamma}\right) \\
G_{\beta_{P-2 \mathrm{D}}}=\frac{1}{2 b^{2} h_{1} \bar{E} \kappa(\gamma)}\left(1+\frac{\beta_{P-2 \mathrm{D}}^{2}}{\gamma}-\frac{\left(1+\beta_{P-2 \mathrm{D}}\right)^{2}}{1+\gamma} c(\gamma)\right)
\end{gathered}
$$

and $\bar{E}=E_{L}$ for plane stress or $\bar{E}=E_{L} /\left(1-v_{L T} v_{T L}\right)$ for plane strain, with $E_{L}$ being the Young's modulus in the longitudinal direction, and $v_{L T}$ and $v_{T L}$ being the in-plane Poisson's ratios. It is worthwhile to note that the influence of the material properties on the ERR is collectively shown by one effective property $\bar{E}$.

Two thickness ratio $\gamma$-dependent correction factors are introduced in Eqs. (13) and (14): A through-thickness shear correction factor $\kappa(\gamma)$ and a pure-mode-II ERR correction factor $c(\gamma)$. It is simple to prove that $\kappa(1 / \gamma)=\kappa(\gamma)$, as required by the physical condition $G_{\theta_{P-2 D}}(1 / \gamma)=G_{\theta_{P-2 D}}(\gamma)$, and that $c(1 / \gamma)=c(\gamma)$, as required by the physical condition $G_{\beta_{P-2 D}}(1 / \gamma)=\left(\beta_{P-2 \mathrm{D}}(\gamma)\right)^{-2} G_{\beta_{P-2 \mathrm{D}}}(\gamma)$. By using FEM simulations with Eqs. (13) and (14), $\kappa(\gamma)$ and $c(\gamma)$ are found to have the following interesting and elegant expressions:

$$
\kappa(\gamma)=0.135535+0.047743 \exp \left(-1.390931 \gamma_{i}^{2}\right)
$$




$$
\begin{gathered}
c(\gamma)=\frac{\left(1+\beta_{P-2 \mathrm{D}}^{2} / \gamma\right)(1+\gamma)}{\left(1+\beta_{P-2 \mathrm{D}}\right)^{2}} C_{F} \\
C_{F}=1-0.070920 \exp \left(-3.280135 \gamma_{i}^{2}\right)
\end{gathered}
$$

It is easily shown that $\kappa(1 / \gamma)=\kappa(\gamma)$ and $c(1 / \gamma)=c(\gamma)$ as required by the physical conditions stated above. They are also shown in Fig. 3. It is interesting to see that $\kappa(\gamma)$ has a perfect normal distribution form with respect to $\gamma_{i}$.

\subsection{Mixed-mode partitions under general loads}

Based on the authors' previous work [1,6-10,21-23], the total ERR under the general loading conditions as shown in Fig. 1a can be written as

$$
\begin{aligned}
& G_{I}=C_{I}\left(M_{1 B}-\frac{M_{2 B}}{\beta_{1-2 \mathrm{D}}}-\frac{N_{1 B e}}{\beta_{2-2 \mathrm{D}}}-\frac{P_{1 B}}{\beta_{3-2 \mathrm{D}}}-\frac{P_{2 B}}{\beta_{4-2 \mathrm{D}}}\right)^{2} \\
& G_{I I}=C_{I I}\left(M_{1 B}-\frac{M_{2 B}}{\theta_{1-2 \mathrm{D}}}-\frac{N_{1 B e}}{\theta_{2-2 \mathrm{D}}}-\frac{P_{1 B}}{\theta_{3-2 \mathrm{D}}}-\frac{P_{2 B}}{\theta_{4-2 \mathrm{D}}}\right)^{2}
\end{aligned}
$$

where

$$
\begin{gathered}
c_{I}=G_{\theta_{1-2 \mathrm{D}}}\left(1-\frac{\theta_{1-2 \mathrm{D}}}{\beta_{1-2 \mathrm{D}}}\right)^{-2}, \quad c_{I I}=G_{\beta_{1-2 \mathrm{D}}}\left(1-\frac{\beta_{1-2 \mathrm{D}}}{\theta_{1-2 \mathrm{D}}}\right)^{-2} \\
G_{\theta_{1-2 \mathrm{D}}}=\frac{6}{b^{2} h_{1}^{3} \bar{E}}\left(1+\frac{\theta_{1-2 \mathrm{D}}^{2}}{\gamma^{3}}-\frac{\left(1+\theta_{1-2 \mathrm{D}}\right)^{2}}{(1+\gamma)^{3}}\right) \\
G_{\beta_{1-2 \mathrm{D}}}=\frac{6}{b^{2} h_{1}^{3} \bar{E}}\left(1+\frac{\beta_{1-2 \mathrm{D}}^{2}}{\gamma^{3}}-\frac{\left(1+\beta_{1-2 \mathrm{D}}\right)^{2}}{(1+\gamma)^{3}}\right)
\end{gathered}
$$

where $N_{1 B e}=N_{1 B}-N_{2 B} / \gamma$. The pure-mode-I $\theta_{1-2 \mathrm{D}}$ mode and the pure-mode-II $\beta_{1-2 \mathrm{D}}$ mode have previously been determined in Ref. [1] by introducing correction factors into the beam-theory-based pure-mode-I and pure-mode-II mechanical conditions. Then the pure-mode-I $\theta_{2-2 D}$ mode and the pure-mode-II $\beta_{2-2 D}$ mode were obtained by using the orthogonality condition that exists between pure modes. Implicit expressions were given in Ref. [1] for the pure-mode-I $\theta_{1-2 \mathrm{D}}$ and $\theta_{2-2 \mathrm{D}}$ modes, and the pure-mode-II $\beta_{1-2 \mathrm{D}}$ and $\beta_{2-2 \mathrm{D}}$ modes. Their explicit expressions are presented here for the easy use of readers with a clearer mechanical interpretation. 


$$
\begin{gathered}
\theta_{1-2 \mathrm{D}}=-\gamma^{2}-\frac{\bar{c}_{\theta}^{2} \gamma^{2}\left(1-\gamma^{2}\right)}{\bar{c}_{\theta}^{2} \gamma^{2}+\phi(1+\gamma)^{2}} \\
\beta_{1-2 \mathrm{D}}=\frac{\gamma^{2}(3+\gamma)}{1+3 \gamma}-\frac{3 \bar{c}_{\theta}^{2} \gamma^{2}\left(1-\gamma^{2}\right)}{(1+3 \gamma)\left[\bar{c}_{\theta}^{2}+\phi(1+3 \gamma)\right]} \\
\theta_{2-2 \mathrm{D}}=-\frac{6}{h_{1}}-\frac{6 \bar{c}_{\theta}^{2} \gamma(1-\gamma)}{h_{1}\left[\bar{c}_{\theta}^{2}\left(1-\gamma+\gamma^{2}\right)+\phi(1+\gamma)^{2}\right]} \\
\beta_{2-2 \mathrm{D}}= \begin{cases}\frac{2(3+\gamma)}{h_{1}(\gamma-1)}+\frac{2 \bar{c}_{\theta}^{2} \gamma}{h_{1} \phi(\gamma-1)} & \text { if } \gamma \neq 1 \\
\infty & \text { if } \gamma=1\end{cases}
\end{gathered}
$$

where

$$
\bar{c}_{\theta} \approx 6 / 5 \quad \text { and } \quad \phi=\bar{c}_{\theta}{ }^{e} \quad \text { with } \quad e=\left[(1+\gamma)^{3} /\left(1+\gamma^{3}\right)\right]^{1 / 2}
$$

In Eqs. (23) to (26), the first term is the corresponding pure mode from Timoshenko beam theory $[6,8,9]$ and the second term is the correction applied for 2D elasticity.

The pure-mode-I modes, $\theta_{3-2 \mathrm{D}}$ and $\theta_{4-2 \mathrm{D}}$, and the pure-mode-II modes, $\beta_{3-2 \mathrm{D}}$ and $\beta_{4-2 \mathrm{D}}$ are now determined as follows: Equating ERR $G_{I}$ ERR in Eq. (10) and Eq. (18) in the pure-mode-I $\theta_{P-2 \mathrm{D}}$ mode where $P_{2 B}=\theta_{P-2 \mathrm{D}} P_{1 B}$ gives

$$
\beta_{3-2 \mathrm{D}}=\frac{\beta_{1-2 \mathrm{D}}\left(1+\beta_{P-2 \mathrm{D}}\right)}{\beta_{P-2 \mathrm{D}}\left(\theta_{1-2 \mathrm{D}}-\beta_{1-2 \mathrm{D}}\right)\left(G_{\theta_{P-2 \mathrm{D}}} / G_{\theta_{1-2 \mathrm{D}}}\right)^{1 / 2}}
$$

Note that in the case of Timoshenko beams, $\beta_{3-\mathrm{T}}=-(3+\gamma)(3 \kappa \mu / \bar{E})^{1 / 2} /\left[h_{1}(1+\gamma)\right]$ where $\kappa=5 / 6$. Equating $G_{I}$ ERR in Eq. (10) and Eq. (18) in the pure-mode-II $\beta_{P-2 \mathrm{D}}$ mode where $P_{2 B}=\beta_{P-2 \mathrm{D}} P_{1 B}$ gives

$$
\beta_{4-2 \mathrm{D}}=-\beta_{P-2 \mathrm{D}} \beta_{3-2 \mathrm{D}}
$$

Note that in the case of Timoshenko beams, $\beta_{4-\mathrm{T}}=-\gamma \beta_{3-\mathrm{T}}$.

Similarly, equating ERR $G_{I I}$ in Eq. (11) and Eq. (19) in the pure-mode-II $\beta_{P-2 \mathrm{D}}$ mode gives

$$
\theta_{3-2 \mathrm{D}}=\frac{\theta_{1-2 \mathrm{D}}\left(1+\beta_{P-2 \mathrm{D}}\right)}{\left(\beta_{1-2 \mathrm{D}}-\theta_{1-2 \mathrm{D}}\right)\left(G_{\beta_{P-2 \mathrm{D}}} / G_{\beta_{1-2 \mathrm{D}}}\right)^{1 / 2}}
$$

Equating $G_{I I}$ ERR in Eq. (11) and Eq. (19) in the pure-mode-I $\theta_{P-2 \mathrm{D}}$ mode gives 


$$
\theta_{4-2 \mathrm{D}}=-\theta_{P-2 \mathrm{D}} \theta_{3-2 \mathrm{D}}
$$

Note that $\theta_{3-\mathrm{T}}=\theta_{4-\mathrm{T}}=-\infty$. Again, it is worth noting that in the context of $2 \mathrm{D}$ elasticity, the influence of the material properties on the ERR is collectively shown by one effective property $\bar{E}$ and that the pure modes are affected only by the geometry. This is in agreement with Hutchinson and Suo [24].

Therefore, as all pure modes are now known, it is now possible to partition the ERR into its individual mode components for any combination of bending moments, axial forces and shear forces by using Eqs. (18) and (19).

\section{Numerical verification}

To verify the present analytical partition theory, a program of 2D FEM simulations was carried out on the DCB shown in Fig. 1a using MSC/NASTRAN. The present analytical theory has previously been verified for combinations of DCB tip bending moments and axial forces only [1] against Suo and Hutchinson's 2D elasticity-based analytical partition theory [4]. Note that although Suo and Hutchinson's theory [4] is regarded as the most accurate for bending moments and axial forces, the method developed in Ref. [1] has a stronger capability for solving more complex mixedmode partition problems, for example, in the bimaterial case [21,22] and in this work which accounts for shear forces.

Hutchinson and Suo [24] showed that the ERR components of an orthotropic material are essentially the same as their isotropic counterparts, except using the longitudinal tensile modulus. For convenience, therefore, isotropic material constants were used, as follows, and apply for all loading conditions and thickness ratios considered in this section: The Young's modulus $E=1000$, the Poisson's ratio $v=0.29$, and the shear modulus $\mu=E /[2(1+v)]$. The DCB geometry consisted of the uncracked length $L=100$, the cracked length $a=10$, the width $b=10$ and the minimum beam thickness $h_{\min }=1$. The thicknesses of the upper and lower beams were therefore dependent on the thickness ratio, $\gamma=h_{2} / h_{1}$, with $h_{1}=h_{\min }$ and $h_{2}=h_{\min }$ if $\gamma>1$, and with $h_{1}=h_{\min } / \gamma$ and $h_{2}=h_{\min }$ if $\gamma<1$. Since the FEM is dimensionless in nature, the model's parameters are given here without units; however, if engineering scale-appropriate units are desired in the following, then units of mm and $\mathrm{N}$ may be chosen for length and force respectively from which the consistent set of units follows.

To verify the present analytical theory, the thickness ratio $\gamma$ was varied in the range $1 / 10 \leq \gamma \leq 10$ under three different sets of loading conditions. In the first loading condition there were only through-thickness shear forces at the crack tip and $P_{2 B}$ was varied in the range 
$-10,000 \leq P_{2 B} \leq 10,000$ with $P_{1 B}=1000$. In the second loading condition there was a combination of through-thickness shear forces and bending moments at the crack tip and $P_{1 B}$ was varied in the range $-10,000 \leq P_{1 B} \leq 10,000$ with $M_{1 B}=1000$. In the third loading condition there were through-

thickness shear forces and axial forces at the crack tip and $N_{1 B e}$ was varied in the range $-10,000 \leq N_{1 B e} \leq 10,000$ with $P_{1 B}=1000$, where $N_{1 B e}=N_{1 B}-N_{2 B} / \gamma$.

The FEM meshing procedure was similar to that used in Ref. [22], which is now summarised. Since very fine meshes were required at the crack tip in order to obtain converged numerical solutions, non-uniform meshes were used in order to avoid excessive computation. 2000 square elements of size $p \times p$ were centered on the crack tip in the $x$-direction, and 100 square elements were centered on the crack tip in the $y$-direction. A mesh size of $p=0.001$ was found to provide mesh independence and was used for all simulations in this section. Beyond the region of uniform element size surrounding the crack tip, elements were allowed to grow at a constant rate of 1.1 in both the $x$ - and $y$-directions up to maximum sizes of 1.0 and 0.1 respectively, after which they remained at these maximum sizes. Very small adjustments were made to the element size growth rate, or to the maximum element size, as appropriate, to satisfy the boundary geometry. Axial forces, $N_{1}$ and $N_{2}$, were applied as point forces to the tips of the upper and lower beams respectively and were uniformly-distributed by area. Bending moments, $M_{1}$ and $M_{2}$, were applied as equal and opposite axial forces to the top and bottom corners of each of the upper and lower beam tips respectively. The upper and lower beams were modelled using quadrilateral plane-strain shell elements. A rigid interface between the upper and lower beams was modelled by 'connecting' the translational degrees of freedom of co-located interface nodes on the upper and lower beams using multi-point constraints; however, at the crack tip, instead of rigidly connecting the crack tip nodes, the interface was modelled with normal and shear point springs. The stiffness of both springs was $k_{s}=b p E_{\mathrm{CT}}$ where $E_{\mathrm{CT}}=10^{10}$, which is the Young's modulus of the interface at the crack tip. The spring stiffness $k_{s}$ was sufficiently high in comparison to $E_{L}$ to simulate brittle interfacial cracking without introducing excessive numerical error. The ERRs were calculated using the virtual crack closure technique (VCCT) and the forces from the crack tip springs [23,25,26]. Contact between the upper and lower surfaces of the crack was not considered.

\subsection{Through-thickness shear forces alone at the crack tip}

This section considers the first loading condition in which there were only through-thickness shear forces at the crack tip and $P_{2 B}$ was varied in the range $-10,000 \leq P_{2 B} \leq 10,000$ with $P_{1 B}=1000$. To achieve this loading condition, DCB tip through-thickness shear forces were applied 
as $P_{1}=P_{1 B}$ and $P_{2}=P_{2 B}$. To avoid bending moments at the crack tip, bending moments were also applied at the DCB tip as $M_{1}=-P_{1} a$ and $M_{2}=-P_{2} a$.

Fig. 4a shows the differences between the total ERRs $G$ from the present analytical theory and from the 2D FEM for each value of $\gamma$ and $P_{2 B} / P_{1 B}$. Fig. 4b shows the differences between the ERR partitions $G_{I} / G$ from the present analytical theory and from the 2D FEM. Note that in Fig. 4, the subscript 'th' notation denotes quantities from the analytical theory whereas the subscript notation 'FEM' denotes quantities from the 2D FEM. There is excellent agreement between the present analytical theory and the 2D FEM results for both the total ERR $G$ and the ERR partition $G_{I} / G$ for the majority of $\gamma$ and $P_{2 B} / P_{1 B}$ values considered. The areas of slightly increased error on both Fig. 4a and Fig. 4b are a result of the small values of the total ERR $G$ in these regions, therefore magnifying the apparent error between the present analytical theory and 2D FEM. To examine this further, Fig. 5 compares the absolute values of $G$ and $G_{I} / G$ from the present analytical theory and the FEM for the cross-sections through Figs. $4 \mathrm{a}$ and $4 \mathrm{~b}$ where $\log _{10}(1 / \gamma)=-0.7$. It is seen that there is excellent agreement for the whole range of $P_{2 B} / P_{1 B}$ and that $G$ becomes small close to $P_{2 B} / P_{1 B}=10$ which is where the error increases in Figs. 4a and 4b.

\subsection{General loading conditions}

In the second loading condition, there was a combination of through-thickness shear forces and bending moments at the crack tip and $P_{1 B}$ was varied in the range $-10,000 \leq P_{1 B} \leq 10,000$ with $M_{1 B}=1000$. To achieve this loading, a DCB tip through-thickness shear force and a DCB tip bending moment were applied as $P_{1}=P_{1 B}$ and $M_{1}=1000-P_{1} a$ respectively.

Fig. 4c shows the differences between the total ERRs $G$ from the present analytical theory and from the 2D FEM for each value of $\gamma$ and $P_{1 B} / M_{1 B}$. Fig. 4d shows the differences between the ERR partitions $G_{I} / G$ from the present analytical theory and from the 2D FEM. There is excellent agreement between the present analytical theory and 2D FEM results for both the total ERR $G$ and the ERR partition $G_{I} / G$ for the majority of $\gamma$ and $P_{1 B} / M_{1 B}$ values considered. It is worth noting that the maximum error in Fig. 4c has been capped to 0.15 in order to make clearer comparisons between the present analytical theory and the 2D FEM. The areas of increased error in Fig. 4c and Fig. $4 \mathrm{~d}$ are also due to the small values of the ERR $G$ in these regions. Fig. 6 compares the absolute values of $G$ and $G_{I} / G$ from the present analytical theory and the FEM for the crosssections through Figs. $4 \mathrm{c}$ and $4 \mathrm{~d}$ where $\log _{10}(1 / \gamma)=0.8$. It is seen that there is excellent agreement 
for the whole range of $P_{1 B} / M_{1 B}$ and that $G$ becomes small close to $P_{1 B} / M_{1 B}=-1$ which is where the error increases in Figs. 4c and $4 \mathrm{~d}$.

Finally, the third loading condition is considered, in which there were through-thickness shear forces and axial forces at the crack tip and $N_{1 B e}$ was varied in the range $-10,000 \leq N_{1 B e} \leq 10,000$ with $P_{1 B}=1000$. To achieve this loading, a DCB tip through-thickness shear force, a DCB tip bending moment, and a DCB tip axial force were applied as $P_{1}=P_{1 B}, M_{1}=-P_{1} a$ and $N_{1}=N_{1 B e}$ respectively.

Fig. 4e shows the differences between the total ERRs $G$ from the present analytical theory and from the 2D FEM for each value of $\gamma$ and $N_{1 B e} / P_{1 B}$. Fig. $4 \mathrm{f}$ shows the differences between the ERR partitions $G_{I} / G$ from the present analytical theory and from the 2D FEM. Again, there is excellent agreement between the present analytical theory and 2D FEM results for both the total ERR $G$ and the ERR partition $G_{I} / G$ for the majority of $\gamma$ and $N_{1 B e} / P_{1 B}$ considered. The maximum error in both Fig. 4e and Fig. 4f has again been capped to 0.15 in order to make clearer comparisons between the present analytical theory and the 2D FEM. The areas of increased error in both Fig. 4e and Fig. 4f are also due to the small values of the ERR $G$ in these regions. Fig. 7 compares the absolute values of $G$ and $G_{I} / G$ from the present analytical theory and the FEM for the cross-sections through Figs. $4 \mathrm{e}$ and $4 \mathrm{f}$ where $\log _{10}(1 / \gamma)=0.9$. It is seen that there is excellent agreement for the whole range of $N_{1 B e} / P_{1 B}$ and that $G$ becomes small close to $N_{1 B e} / P_{1 B}=-1$ which is where the error increases in Figs. 4e and 4f.

\section{Conclusions}

The authors' existing analytical partition theory for mixed-mode fractures in 2D elastic orthotropic laminated beams with rigid interfaces [1] has been successfully extended to account for through-thickness shear forces. Results for the present analytical partition theory have been compared to those from the 2D FEM and excellent agreement has been observed, particularly when the total ERR $G$ is not close to zero. This work now offers a means of calculating the 2D elasticitybased ERR partition for an orthotropic laminated DCB under any combination of bending moments, axial forces and shear forces.

The present analytical partition theory is achieved by introducing correction factors into the authors' Timoshenko beam partition theory [6-10]. The correction factors were determined by using the 2D FEM. It is interesting that the correction factors closely follow elegant normal distributions around a symmetric DCB geometry. 


\section{References}

[1] Harvey CM, Wood JD, Wang S, Watson A. A novel method for the partition of mixed-mode fractures in 2D elastic laminated unidirectional composite beams. Composite Structures 2014;116:589-594.

[2] Lu TJ, Xia C, Hutchinson JW. Delamination of beams under transverse shear and bending. Material Science and Engineering 1994;A188:103-112.

[3] Wang J, Qiao Z. Interface crack between two shear deformable elastic layers. Journal of the Mechanics and Physics of Solids 2004;52:891-905.

[4] Suo Z, Hutchinson JW. Interface crack between two elastic layers. International Journal of Fracture 1990;43:1-18.

[5] Li S, Wang J, Thouless MD. The effects of shear on the delamination in layered materials. Journal of the Mechanics and Physics of Solids 2004;52:193-214.

[6] Wang S, Harvey CM. Mixed mode partition theories for one dimensional fracture. Engineering Fracture Mechanics 2012;79:329-352.

[7] Harvey CM, Wang S. Mixed-mode partition theories for one-dimensional delamination in laminated composite beams. Engineering Fracture Mechanics 2012;96:737-759.

[8] Harvey CM, Wang S. Experimental assessment of mixed-mode partition theories. Composite Structures 2012;94:2057-67.

[9] Harvey CM, Eplett MR, Wang S. Experimental assessment of mixed-mode partition theories for generally laminated composite beams. Composite Structures 2015;124:10-18.

[10] Wang S, Harvey CM, Wang B, Watson A. Post-local buckling-driven delamination in bilayer composite beams. Composite Structures 2015;133:1058-66.

[11] Davidson BD, Fariello PL, Hudson RC, Sundararaman V. Accuracy assessment of the singular-field-based mode-mix decomposition procedure for the prediction of delamination. In: Hooper SJ, editor. Composite materials: testing and design (thirteenth volume), ASTM STP 1242. American Society for Testing and Materials, 1997, pp. 109-128.

[12] Davidson BD, Gharibian SJ, Yu LJ. Evaluation of energy release rate-based approaches for predicting delamination growth in laminated composites. International Journal of Fracture 2000;105:343-365.

[13] Davidson BD, Bialaszewski RD, Sainath SS. A non-classical, energy release rate based approach for predicting delamination growth in graphite reinforced laminated polymeric composites. Composites Science and Technology 2006;66:1479-1496. 
[14] Hashemi S, Kinloch AJ, Williams JG. The analysis of interlaminar fracture in uniaxial fibrepolymer composites. Proceeding of the Royal Society A 1990;427:173-199.

[15] Kinloch AJ, Wang Y, Williams JG, Yayla P. The mixed-mode delamination of fibre composite materials. Composites Science and Technology 1993;47:225-237.

[16] Charalambides M, Kinloch AJ, Wang Y, Williams JG. On the analysis of mixed-mode failure. International Journal of Fracture 1992;54:269-291.

[17] Hashemi S, Kinloch AJ, Williams G. Mixed-mode fracture in fiber-polymer composite laminates. In: O’Brien TK, editor. Composite materials: fatigue and fracture (third volume), ASTM STP 1110. Philadelphia, PA: American Society for Testing and Materials, 1991. pp. 143-168.

[18] Conroy M, Sørensen BF, Ivankovic A. Combined numerical and experimental investigation of mode-mixity in beam like geometries. In: Proceedings of the 37th Annual Meeting of the Adhesion Society, Feburary 2014, San Diego, California, USA.

[19] Kutlu Z, Chang FK. Composite panels containing multiple through-the-width delaminations and subjected to compression. Part I: analysis. Composite Structures 1995;31:273-296.

[20] Kutlu Z, Chang FK. Composite panels containing multiple through-the-width delaminations and subjected to compression: Part II: experiments and verification. Composite Structures 1995;31:297-314.

[21] Harvey CM, Wood JD, Wang S. Brittle interfacial cracking between two dissimilar elastic layers: Part 1 - Analytical development. Composite Structures 2015;134:1076-1086.

[22] Harvey CM, Wood JD, Wang S. Brittle interfacial cracking between two dissimilar elastic layers: Part 2 - Numerical verification. Composite Structures 2015;134:1087-1094.

[23] Harvey CM, Wang S. Numerical and analytical study of delamination in composite laminates. International Journal of Engineering Systems Modelling and Simulation 2012;4:120-37.

[24] Hutchinson JW, Suo Z. Mixed mode cracking in layered Materials. Advances in Applied Mechanics 1992;92:63-191.

[25] Zhang Y, Wang S. Buckling, post-buckling and delamination propagation in debonded composite laminates: Part 1 theoretical development. Composite Structures 2009;88:121-30.

[26] Wang S, Zhang Y. Buckling, post-buckling and delamination propagation in debonded composite laminates: Part 2 numerical applications. Composite Structures 2009;88:131-46. 


\section{List of Figures}

Fig. 1: A laminated DCB. (a) General description. (b) Details local to the crack tip.

Fig. 2: Variation of the 2D elasticity theory-based pure mode II $\beta_{P \text {-2D }}$ for through-thickness shear forces only with respect to $\gamma$ using FEM.

Fig. 3: Variation of the shear correction factor $\kappa(\gamma)$ from Eq. (15) and the FEM, and variation of the correction factor $c(\gamma)$ from Eq. (16) and the FEM, both with respect to $\gamma$.

Fig. 4: Comparison of the present analytical theory and the 2D FEM for the total ERR $G$ and the ERR partition $G_{I} / G$ for variable $\gamma$ and loading conditions $P_{2 B} / P_{1 B}, P_{1 B} / M_{1 B}$ and $N_{1 B e} / P_{1 B}$.

Fig. 5: Comparison of the present analytical theory and the 2D FEM for the total ERR $G$ and the ERR partition $G_{I} / G$ for $\log _{10}(1 / \gamma)=-0.7$ and variable $P_{2 B} / P_{1 B}$.

Fig. 6: Comparison of the present analytical theory and the 2D FEM for the total ERR $G$ and the ERR partition $G_{I} / G$ for $\log _{10}(1 / \gamma)=0.8$ and variable $P_{1 B} / M_{1 B}$.

Fig. 7: Comparison of the present analytical theory and the 2D FEM for the total ERR $G$ and the ERR partition $G_{I} / G$ for $\log _{10}(1 / \gamma)=0.9$ and variable $N_{1 B e} / M_{1 B}$. 


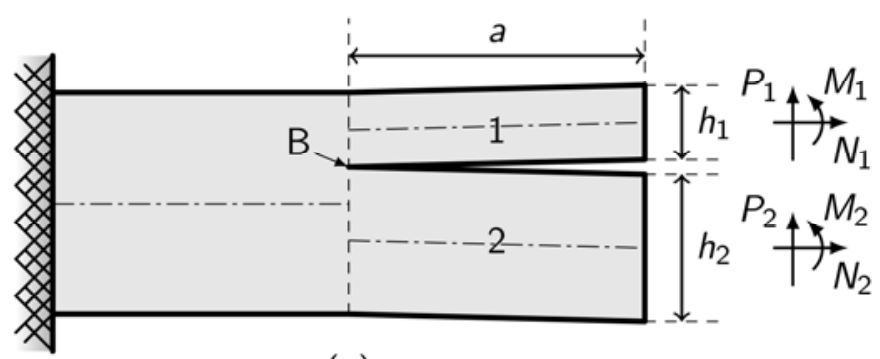

(a)

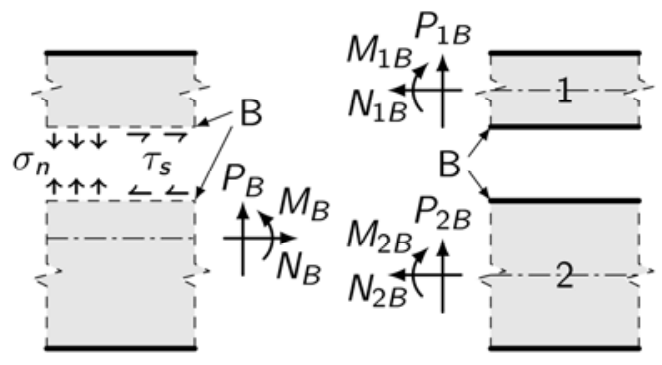

(b)

Fig. 1: A laminated DCB. (a) General description. (b) Details local to the crack tip. 


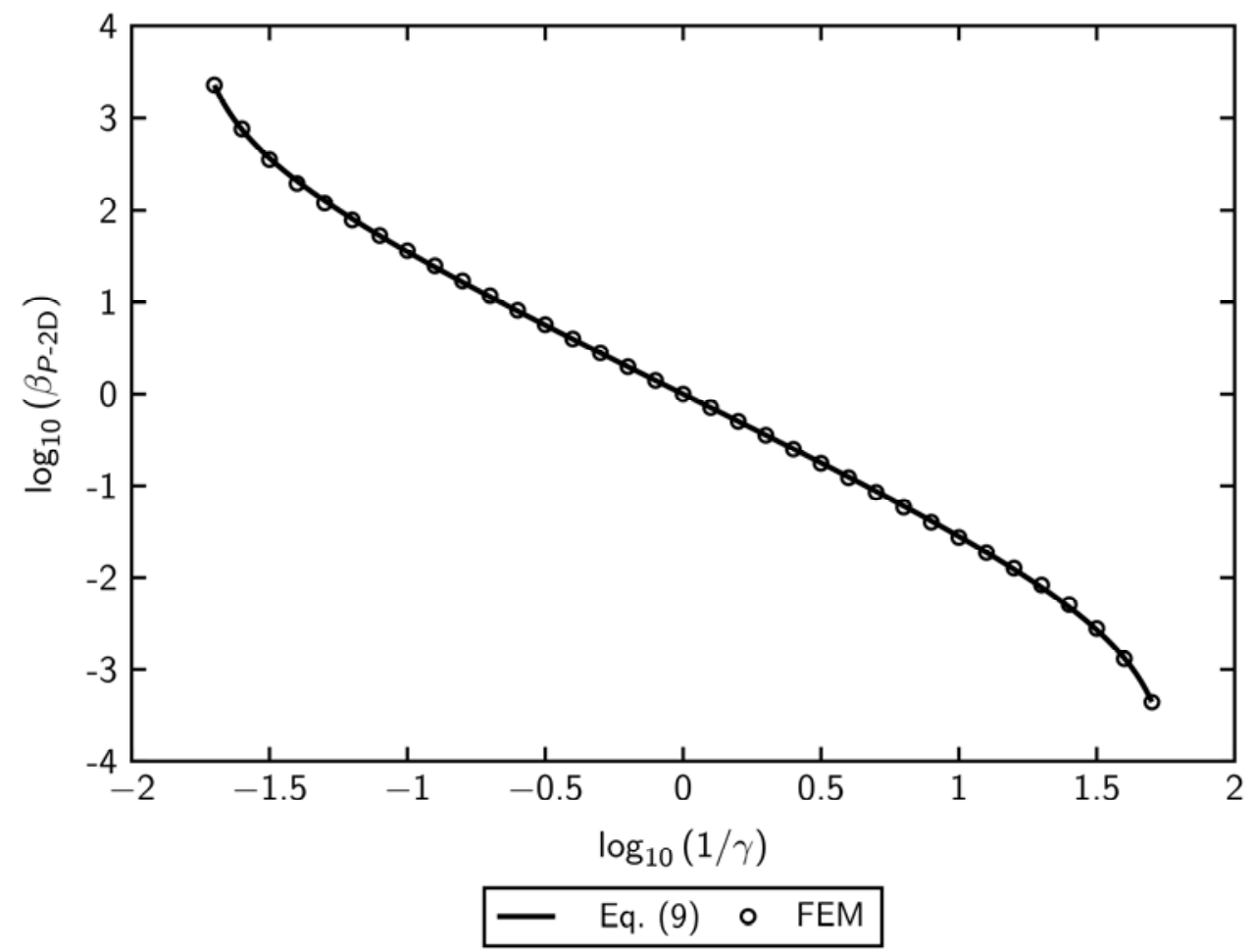

Fig. 2: Variation of the 2D elasticity theory-based pure mode II $\beta_{P-2 D}$ for through-thickness shear forces only from Eq. (9) and the 2D FEM with respect to $\gamma$. 


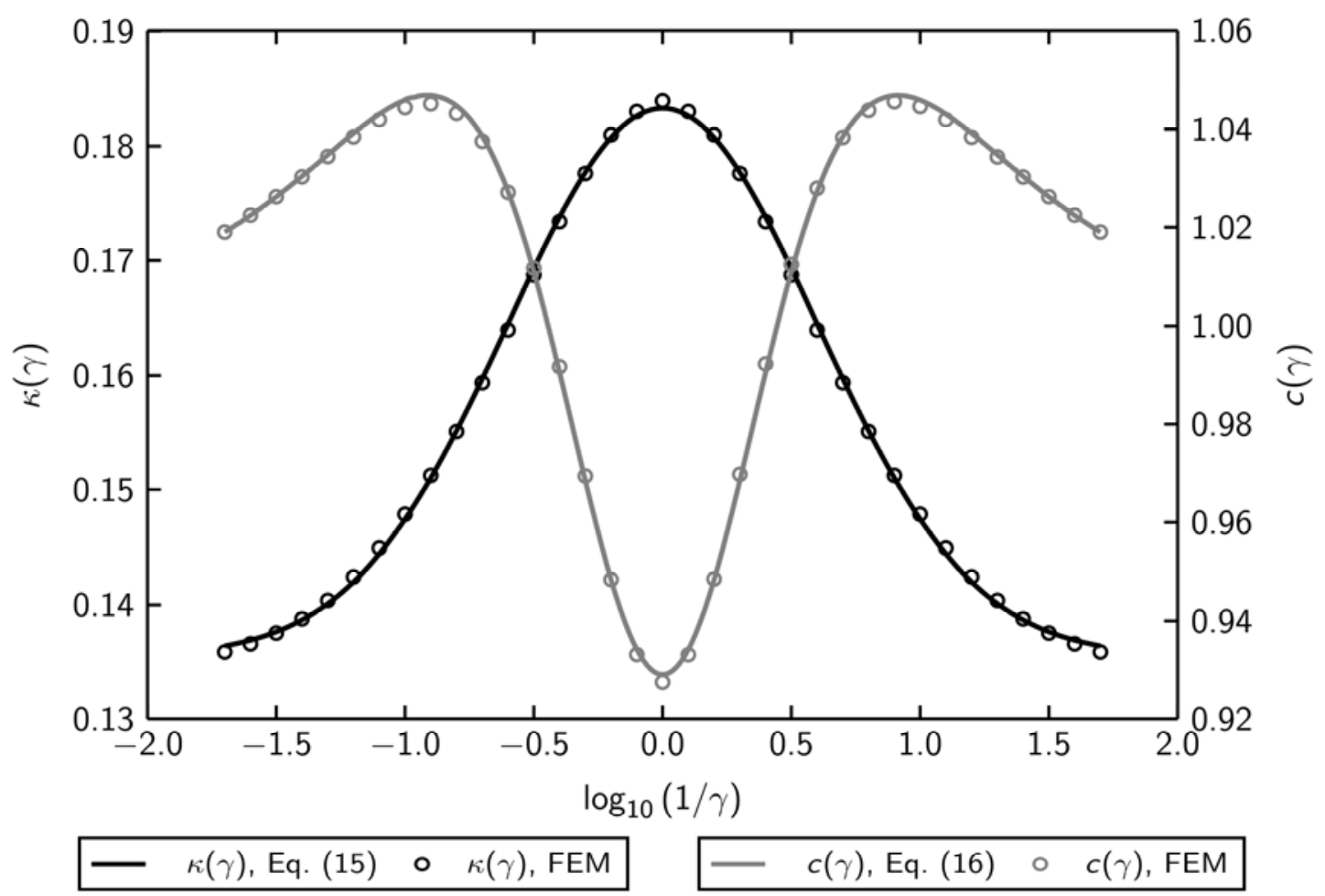

Fig. 3: Variation of the shear correction factor $\kappa(\gamma)$ from Eq. (15) and the 2D FEM, and variation of the correction factor $c(\gamma)$ from Eq. (16) and the 2D FEM, both with respect to $\gamma$. 
(a) $\left|1-G_{\text {th }} / G_{\mathrm{FEM}}\right|$

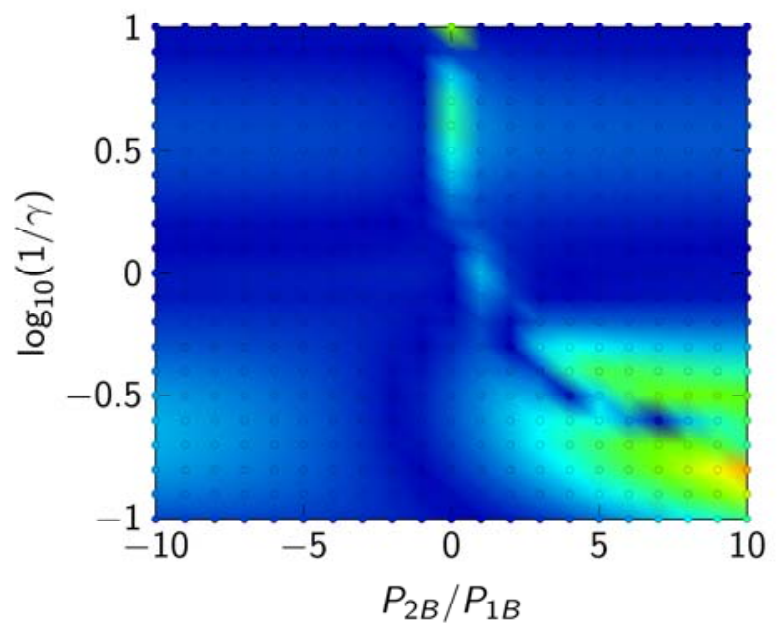

(c) $\left|1-G_{\mathrm{th}} / G_{\mathrm{FEM}}\right|$

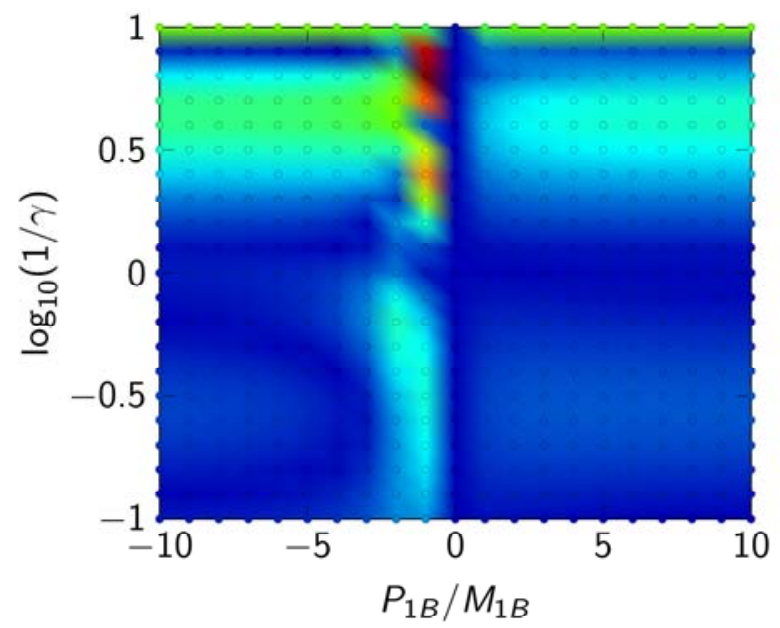

(e) $\left|1-G_{\text {th }} / G_{\text {FEM }}\right|$
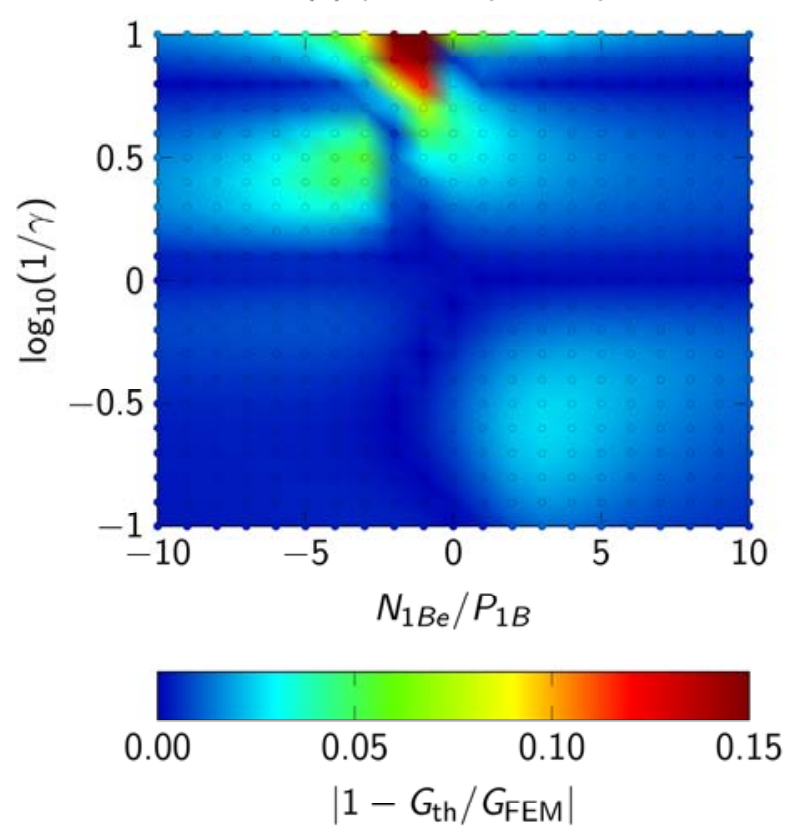

(b) $\left|\left(G_{l} / G\right)_{\text {th }}-\left(G_{l} / G\right)_{\text {FEM }}\right|$

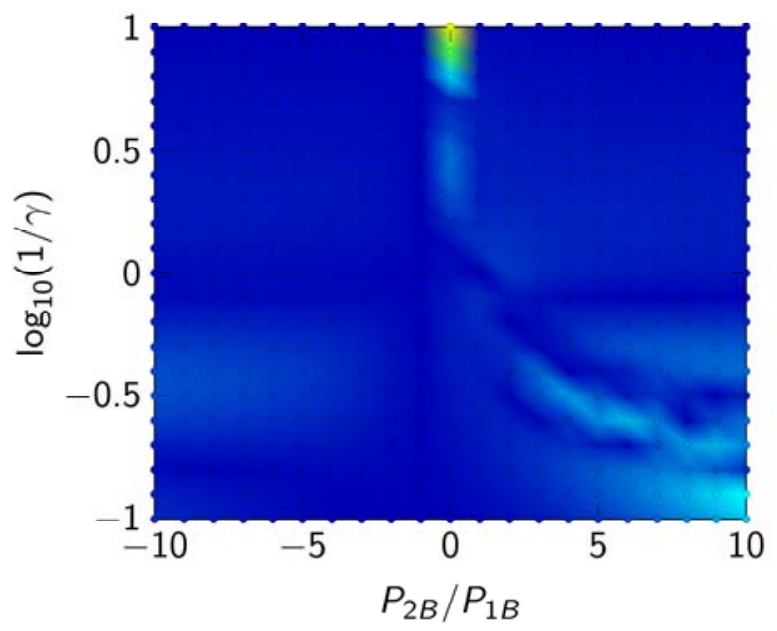

(d) $\left|\left(G_{l} / G\right)_{\mathrm{th}}-\left(G_{l} / G\right)_{\mathrm{FEM}}\right|$

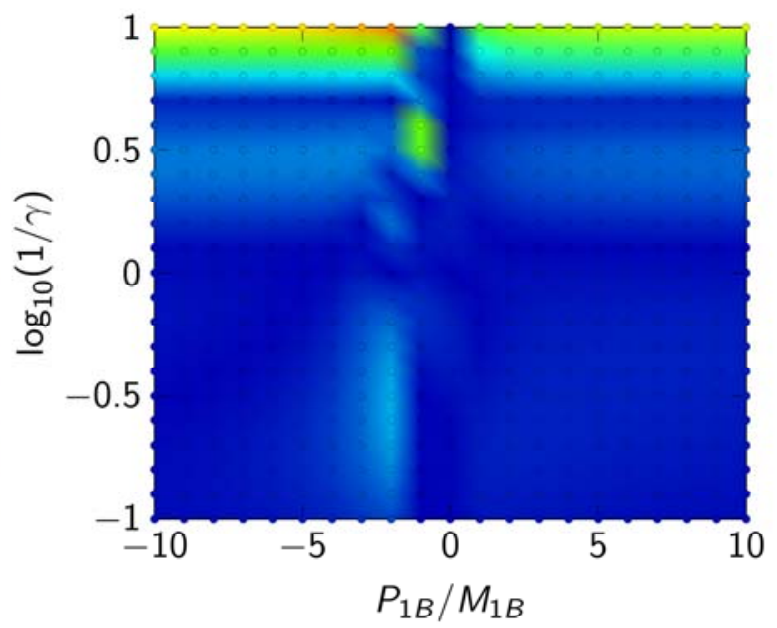

(f) $\left|\left(G_{l} / G\right)_{\text {th }}-\left(G_{l} / G\right)_{\text {FEM }}\right|$

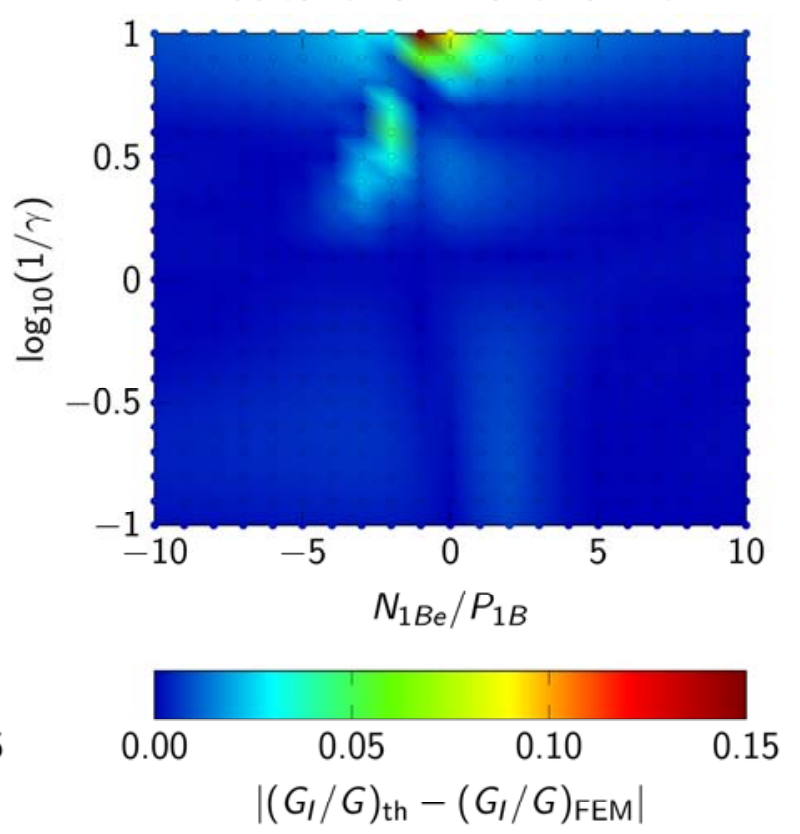

Fig. 4: Comparison of the present analytical theory and the 2D FEM for the total ERR $G$ and the ERR partition $G_{I} / G$ for variable $\gamma$ and loading conditions $P_{2 B} / P_{1 B}, P_{1 B} / M_{1 B}$ and $N_{1 B e} / P_{1 B}$. 
(a) $\left|1-G_{\text {th }} / G_{\text {FEM }}\right|$

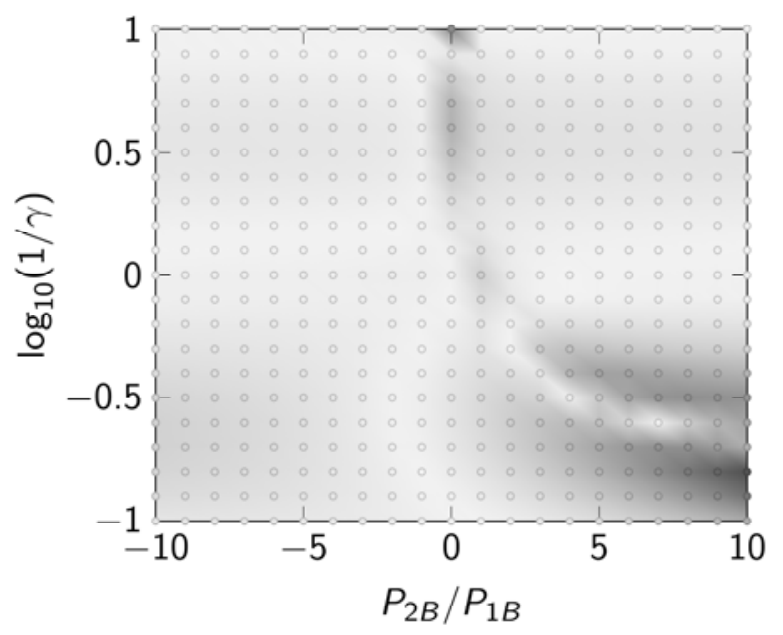

(c) $\left|1-G_{\text {th }} / G_{\text {FEM }}\right|$

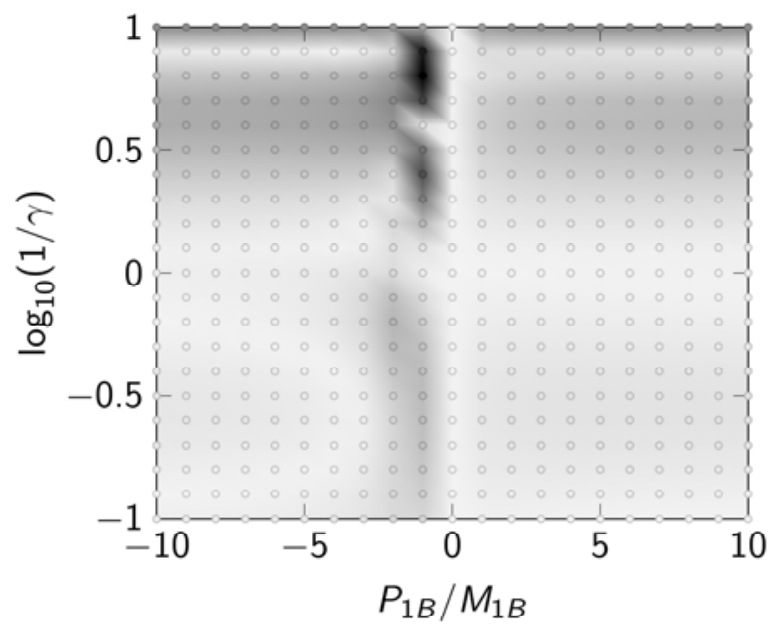

(e) $\left|1-G_{\text {th }} / G_{\text {FEM }}\right|$
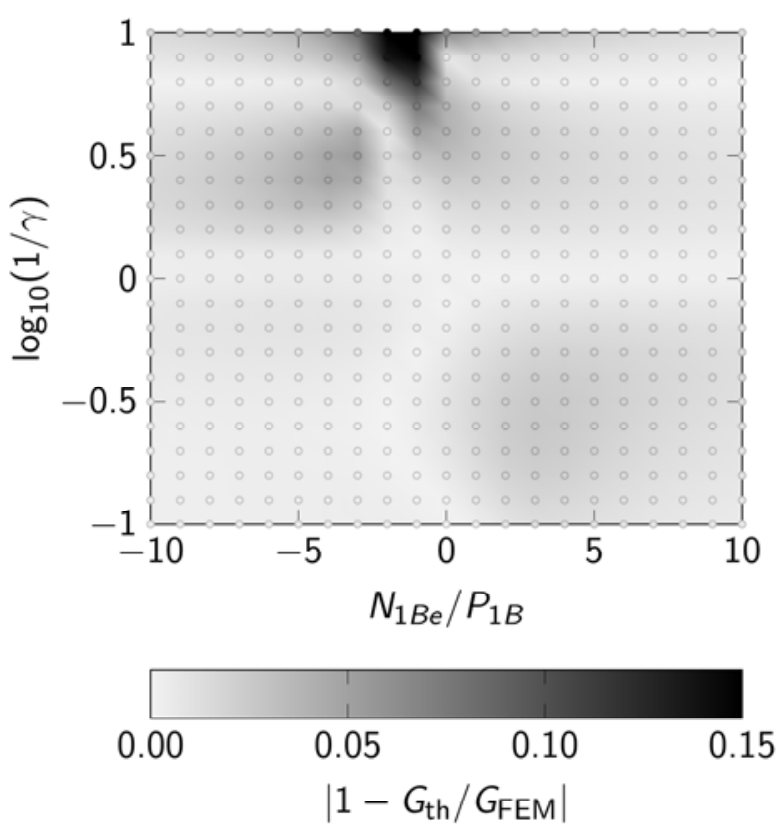

(b) $\left|\left(G_{l} / G\right)_{\mathrm{th}}-\left(G_{l} / G\right)_{\mathrm{FEM}}\right|$

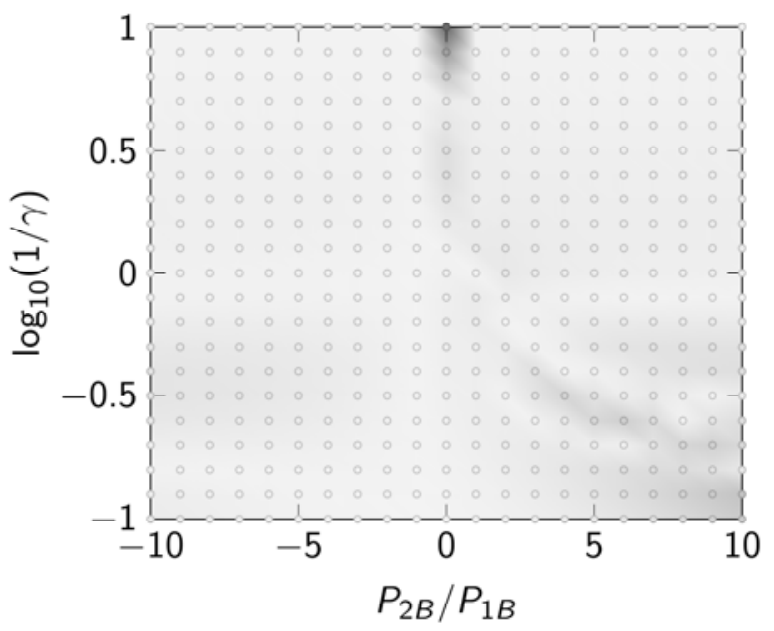

(d) $\left|\left(G_{l} / G\right)_{\mathrm{th}}-\left(G_{l} / G\right)_{\mathrm{FEM}}\right|$

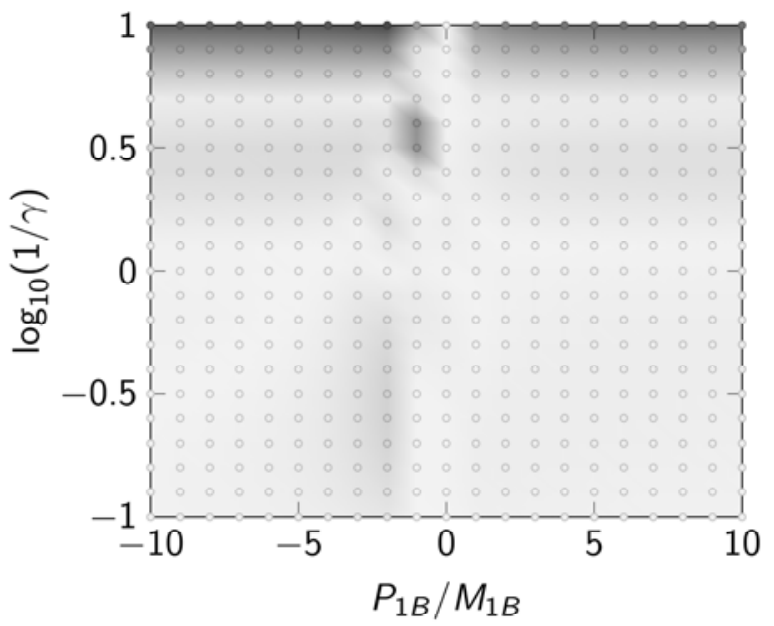

(f) $\left|\left(G_{l} / G\right)_{\text {th }}-\left(G_{l} / G\right)_{\text {FEM }}\right|$

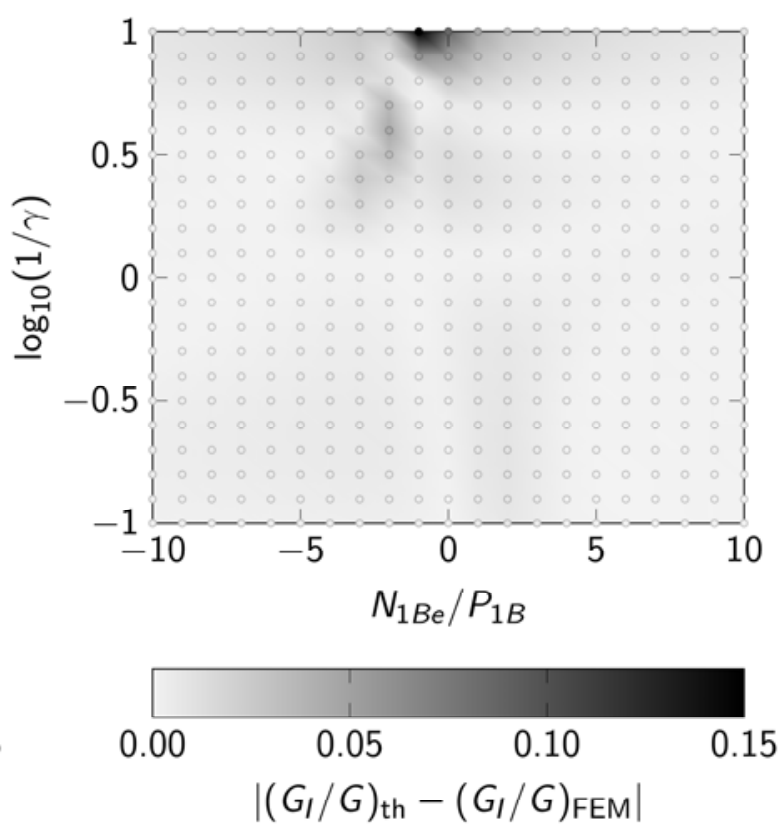

Fig. 4: Comparison of the present analytical theory and the 2D FEM for the total ERR $G$ and the ERR partition $G_{I} / G$ for variable $\gamma$ and loading conditions $P_{2 B} / P_{1 B}, P_{1 B} / M_{1 B}$ and $N_{1 B e} / P_{1 B}$. 


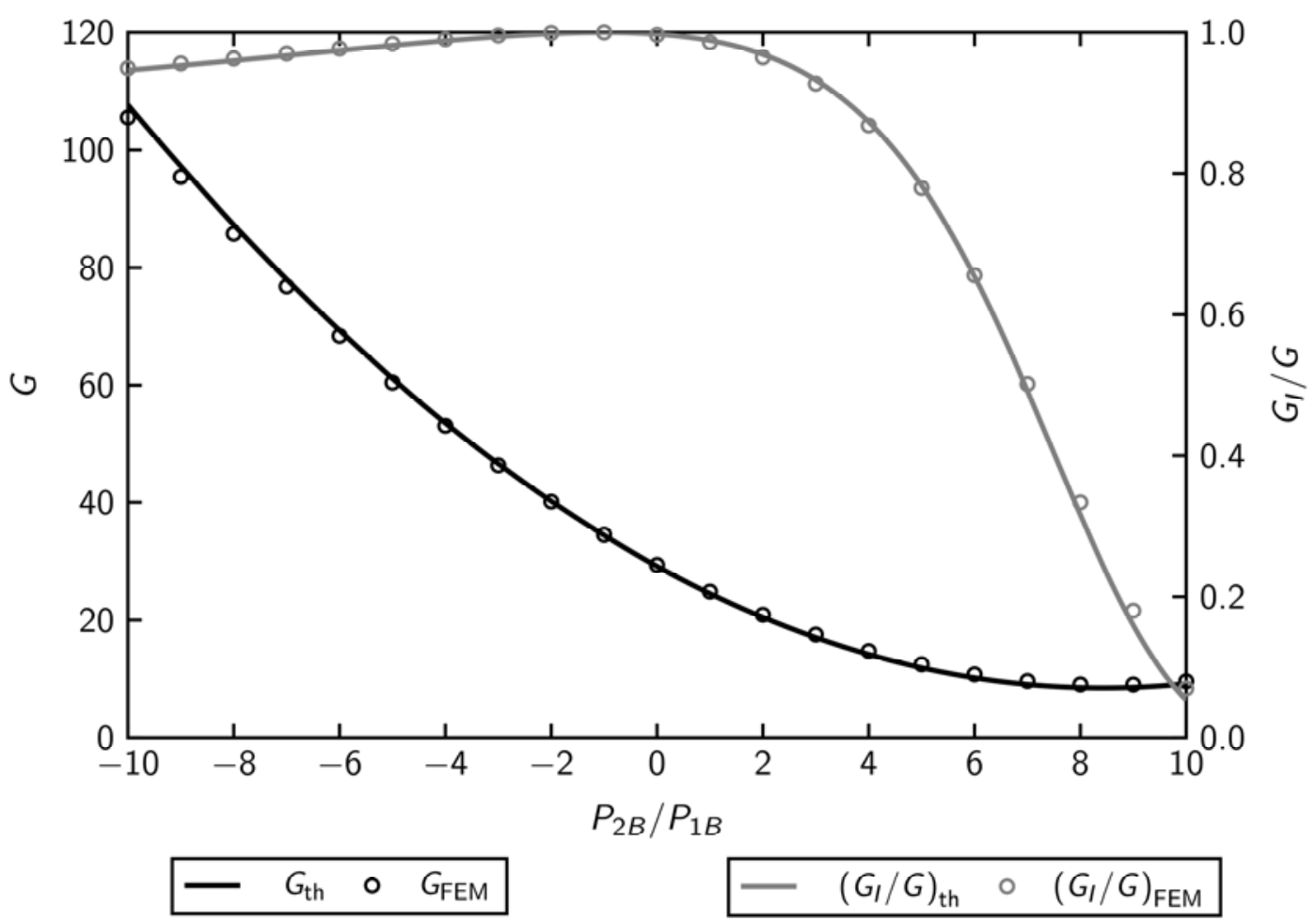

Fig. 5: Comparison of the present analytical theory and the 2D FEM for the total ERR $G$ and the ERR partition $G_{I} / G$ for $\log _{10}(1 / \gamma)=-0.7$ and variable $P_{2 B} / P_{1 B}$. 


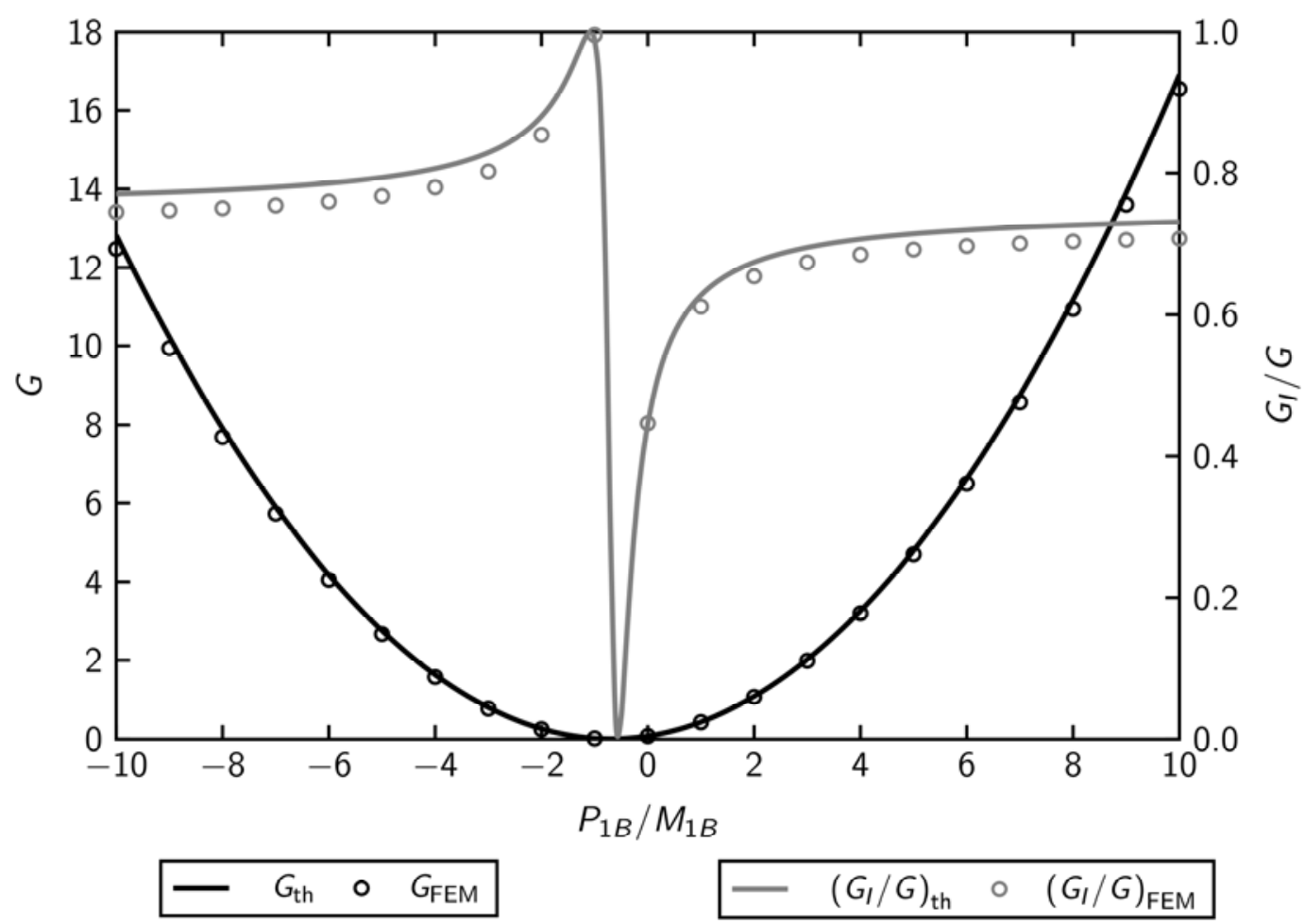

Fig. 6: Comparison of the present analytical theory and the 2D FEM for the total ERR $G$ and the ERR partition $G_{I} / G$ for $\log _{10}(1 / \gamma)=0.8$ and variable $P_{1 B} / M_{1 B}$. 


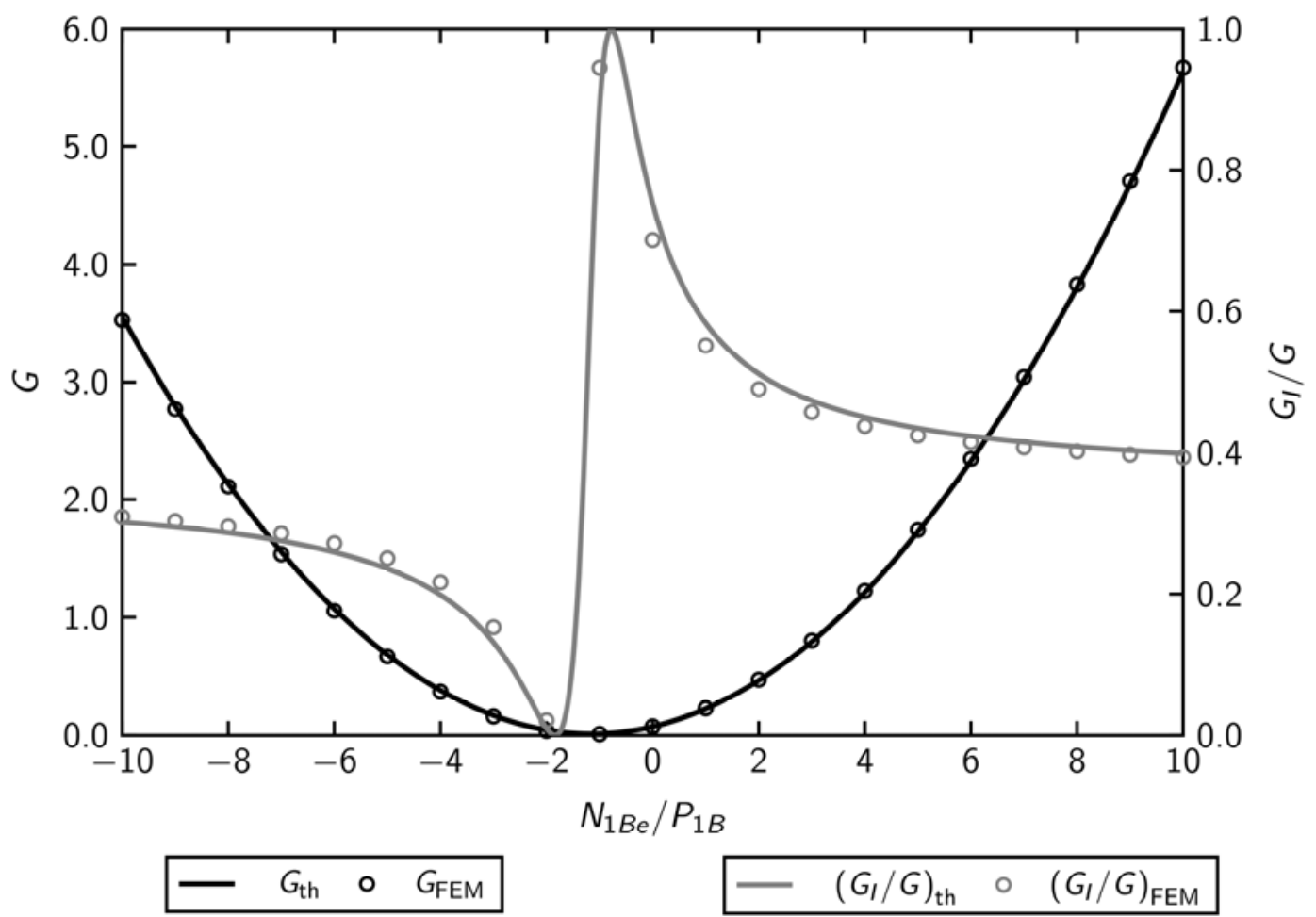

Fig. 7: Comparison of the present analytical theory and the 2D FEM for the total ERR $G$ and the ERR partition $G_{I} / G$ for $\log _{10}(1 / \gamma)=0.9$ and variable $N_{1 B e} / P_{1 B}$. 